\title{
Editorial: International Conference on Fatigue Damage of Structural Materials
}

The tenth biennial International Conference on Fatigue Damage of Structural Materials was held in Hyannis, Massachusetts from the 21-26 September 2014. This prestigious and long running conference brought together 130 delegates from 28 countries around the world to discuss how to characterize, predict and analyse the fatigue damage of structural materials. The conference was covering following themes:

Structural metals and alloys pertinent to the aerospace, marine,off-shore, power generation and land based transportation industries.

- Novel experimental methods to characterise fatigue damage and crack growth.

- Overload/underload, arbitrary loading sequences, service spectrum loads, combined HCF/LCF.

- Residual stress effects on fatigue damage and crack growth, measurement of internal stresses.

- Extreme environments, including the effects of corrosion, oxidation, abrasion, elevated or cryogenic temperatures.

- Innovative theoretical approaches, computational and analytical methods.

- Life prediction methodologies for structural metals and alloys.

- Fatigue mechanisms in advanced alloys and metallic systems.

This special issue is a collection of 23 out of 54 oral presentations and 67 poster presentations that were selected amongst the numerous abstracts proposed to be presented into 15 single oral sessions and 3 poster sessions.

Marie-Christine Baietto

INSA Lyon, 20 Av. A. Einstein, Batiment J. d'Alembert, 69621 Villeurbanne, France.

James Burns

Department of Materials Science \& Engineering, University of Virginia, PO Box 400745, 395 McCormick Road, Charlottesville, VA 22904-4745, United States

Office: Wilsdorf Hall Room 318, United States

David Nowell

Department of Engineering Science, University of Oxford, Parks Road, Oxford OX1 3PJ, United Kingdom

Sylvie Pommier

Laboratoire de mécanique et technologie (LMT-Cachan), 61, avenue du président Wilson, 94235 Cachan cedex, France

Manuela Sander

Universität Rostock, Fakultät für Maschinenbau, und Schiffstechnik, 
Lehrstuhl für Strukturmechanik, 18051 Rostock, Germany

Mark Whittaker

College of Engineering, Swansea University, Singleton Park, Swansea SA2 8PP, United Kingdom 